\title{
Historical aspects in central vestibular syndrome
}

\author{
Miorita Toader', Alina Oprea', Mircea Draghici², Andrei Osman³, Carmen Mogoanta ${ }^{4}$ \\ 1"Grigore Alexandrescu" Emergency Clinical Hospital for Children, Bucharest, Romania \\ 2Dentirad Hospital, Ploiesti, Romania \\ ${ }^{3}$ Regional Clinical Hospital, Craiova, Romania \\ ${ }^{4}$ University of Medicine and Pharmacy, Craiova, Romania
}

\begin{abstract}
Central vestibular syndromes raise many challenges through their differential diagnosis. Throughout the last two centuries the advent of medical technologies provided new insights into the underlying pathophysiological mechanisms. The first studies date back in 1824 when Flourens researched the physiology of the labyrinth. As a result of the incomplete knowledge on the anatomy of central vestibular structures, the cerebellum was initially incriminated for the vestibular syndromes. First similarities between labyrinth related symptoms and cerebellum disorders were investigated by Babinski in 1913. Eventually, vestibular signs were also attributed to cerebellar peduncles lesions. The article outlines a chronological systematization of the steps taken throughout time in establishing the features of central vestibular syndrome.
\end{abstract}

Keywords: central vestibular syndrome, vestibular system, history

\section{THE BEGINNING OF THE 20 ${ }^{\text {TH }}$ CENTURY}

For almost a century, starting with Flourens's research in 1824 on the physiology of the peripheral labyrinth, the cerebellum was considered responsible for the etiology of vestibular syndromes. He was the first one to pioneer the use ablation studies for determining the functions of vestibular structures and cerebellum (1). In 1913, Babinski sustained that the cerebellum is involved like the labyrinth in the genesis of vertigo or nystagmus (2). Flourens and Magendie have published papers showing the presence of similar symptoms in the presentation both of semicircular channels lesion and cerebellar peduncles lesions (3).

An important contribution came from researchers in Netherlands. Between 1911-1914, Leidler and Bauer have performed various experimental studies. Through selective pons lesions they have highlighted the importance of central vestibular pathways. Furthermore, by interrupting the arciform fibers which connect the vestibular nuclei with posterior longitudinal fasciculus, they obtained the same symptoms occurring in vestibular nerve lesions. Lesions pertaining to the knee of the facial nerve produced horizontal or rotating nystagmus on the counter lateral origin of the lesion, rotation of the body on the ipsilateral part of the lesion and tonic deviation of the head. Lesions below the knee induced ipsilateral nistagmus and head deviation (4).

The interdependence between the origin of the lesion and the vestibular signs were also illustrated by Van Gehuchten (5). More than that, based on the research of Flourens and Magendie, Thomas demonstrated that vestibular signs are a strict consequence of lesions in the vestibular central pathways $(3,6)$. The presence of vertigo was strongly correlated with vestibular pathways involvement, regardless of the presence of a cerebellar pathology. Afterwards, in 1929, Alfandari proposed that vertigo is actually a vestibular reflex. In his experiments, vermis removal induced aberrant ocular 
movement only after affecting the roof of fourth ventricle (6).

\section{THE 30'S - NEW CLINICAL BREAKTHROUGHS}

The initiator of the next era in defining central vestibular syndrome was Barré. His main struggle was to define the underlying physiopathological mechanisms. His theory was that the cerebellum was able to entirely influence the type of the vestibular nystagmus. He defined the vestibular nystagmus of the cerebellum as a slow prolonged ocular saccade followed by a rapid fast one. He thoroughly studied the profound interconnections between the cerebellum and vestibular system. Lesions involving both structures lead to the coexistence of vestibular and cerebellar syndromes (7).

$\mathrm{He}$ attributed the disharmony of the vestibular syndrome to the strong connections established between the origin of the central lesions and clinical expressions. Therefore, while in medulla, lesions the nystagmus is horizontal, in cerebral peduncles is both horizontal and vertical, expressing an extensive lesion. Disharmony is also sustained by the fact that slow ocular oscillation occur in different directions (6).

He introduced the concept of "hemiavestibulia" similar nowadays tot the concept of hemianopsia. Unilateral lesions developed different clinical types of nystagmus. Each lesion could block either voluntary or reflex ocular movements on the affected side. He documented his findings in experimental studies through complex instrumental stimulation maneuvers in which he demonstrated the impossibility of generating movements on the ipsilateral direction of the lesion $(7,8)$.

In the same period, Dow and Larsell proved through animal experiments that selective extirpation of the nodular lobe was followed by equilibrium disorders similar to those secondary to vestibular lesions $(9,10)$. The implication of the vermis in equilibrium disorders was later confirmed by cerebellum medulloblastoma. Vermis atrophia affecting the antero-dorsal part clinically expresses as static anomalies associated with a vestibular equilibrium disorder (11).

\section{THE 60'S - THE IMPLEMENTATION OF NYSTAGMOGRAPHY}

An important step towards understanding the complexity of the central vestibular syndrome is represented by the wide implementation of an innovatory diagnostic technique - the nystagmography intended to be a graphic representation of eye movements (12). Studies in the previous years have outlined the necessity for electrical recording in order to accurately describe the nystagmus in vestibular disease. It was based on analyzing the consequences of corneo-retinal potential on the ocular movements. It offered the possibility of measuring the amplitude, frequency and velocity of the slow component (14).

In 1965, Gabersek describes the following nystagmographic features in central disorders. Monocular derivations outline a nystagmus with different frequencies between the two eyes and different speeds for the rapid phases of provoked nystagmus. Binocular derivations show normal nystagmus duration and amplitude with irregular responses and numerous pauses (15).

In 1966, Montandon performed nystagmographic studies on central vestibular lesions. He demonstrated the specific irregular rhythm patterns which interfere with tahinystagmic saccades (16). In 1969 , Pialoux showed abnormal ocular movements after changes in sight directions. The movements correlated with saccadic findings on the nystagmography (17). In the same year, Rubin pointed out the advantages of nystagmography in discovering nystagmus with eyes closed (8).

\section{THE 80'S - DIFFERENTIAL BETWEEN CENTRAL AND PERIPHERAL LESIONS}

Starting in the 70's and culminating in the 80's, the differential diagnosis between central and peripheral vestibular syndrome became more and more accurate. The gold standard was provided by electronystagmography which was used not only as diagnostic tool, but also as a monitoring one. Various stimuls were used to generate nystagmus. While some of them produced normal vestibular syndromes, others generated imbalances in the vestibular system. Therefore, new types of nystagmus were more accurately described: spontaneous, positional, gaze, optokinetic. Specific electronystagmographic features oriented towards a central or peripheral lesion (18).

In central lesions, spontaneous nystagmus follows a vertical or horizontal direction, lacking the rotatory component and bot being inhibited by fixation. Gaze invoked nystagmus will be enhanced by fixation and reduced by eyes closure in central pathology, while in peripheral lesions it will exhib- 
it opposite traits. Positional nystagmus not inhibited by fixation is suggestive for central pathology. Asymmetrical recordings of the slow phase in each direction for optokinetic nystagmus are suggestive for central lesions (18).

During this period, the major drawback of the electronystagmography was its inability to locate the specific anatomic lesion. Therefore, its first-line use was to draw the differential between vestibular peripheral lesions and centrally located lesions. The final probable diagnosis was established after integrating the results of clinical examination and audiological studies (18).

\section{THE $21^{\text {ST }}$ CENTURY - THE ADVENT OF IMAGING TECHNIQUES}

The advent of imaging techniques (computed tomography, magnetic resonance imaging) have paved the way for a new era in the diagnosis of central vestibular syndrome. Brain imaging has earned a pivotal role in the diagnosis of central etiologies such as: multiple sclerosis, brainstem ischemia, Chiari malformation, cerebellar infarction, cerebellar hemorrhage, vestibular schwannoma (19). Before recommending imaging techniques, a corroboration between history and clinical findings must be performed in order to raise suspicion for a central cause (20).

The greatest contribution is in the case of an acute onset of sustained vertigo when a distinction must be made between an acute vascular event or a vestibular neuritis. The necessity is sustained by the presence of neurological signs or symptoms, of risk factors for stroke, of nystagmus with central features (exhibits any direction, is not suppressed by visual fixation, accompanied by severe instability) or a sudden intense progressive headache $(19,20)$.

\section{REFERENCES}

1. Yildirim F.B., Sarikcioglu L. Marie Jean Pierre Flourens (17941867): an extraordinary scientist of his time. Journal of Neurology, Neurosurgery, and Psychiatry. 2007; 78(8):852. doi:10.1136/ jnnp.2007.118380.

2. McGlynn S.M., Schacter D.L. Unawareness of deficits in neuropsychological syndromes J Clin Exp Neuropsychol. 1989 Mar; 11(2):143-205

3. Tizard B. Theories of brain localization from Flourens to Lashley. Med Hist 19593132-145.145

4. Spiegel E.A., Scala N.P. Vertical nystagmus following lesions of the cerebellar vermis. Arch ophthalmol. 1941; 26(4):661-669.

5. Nyberg-Hansen R. Origin and termination of fibers from the vestibular nuclei descending in the medial longitudinal fasciculus. An
Magnetic resonance imaging is recommended for the diagnosis of hemorrhage, infarction, tumor and multiple sclerosis. Whenever, it is not available, the next option should be computed tomography with fine cuts, especially when there is a suspicion for a vascular event involving the cerebellum. For the investigation of etiologies concerning the vertebrobasilar system, magnetic resonance angiography and computed tomography angiography are also recommended $(19,20)$.

Nowadays, the future belongs to functional brain imaging whose main purpose is to decipher the intriguing multisensory and sensorimotor functions of vestibular system. Brain activation studies on humans have unveiled the complex interconnections of vestibular structures with other sensorial structures and the functional consequences of particular peripheral or central vestibular lesions (21).

Actually, signs and symptoms of vestibular disorders can be correlated with the patterns of spontaneous activity during brain imaging. Functional magnetic resonance imaging (fMRI) and positron emission tomography (PET) have demonstrated specific activation-deactivation patterns of the vestibular network in case of acute unilateral peripheral and central vestibular lesions such as vestibular neuritis, bilateral vestibular failure, Wallenberg's syndrome, posterolateral thalamic infarctions (21).

\section{CONCLUSIONS}

Central vestibular syndromes have incited a great amount of research interest in the last two centuries. The chronological evolution of their understanding only convey the difficulties encountered in performing the challenging transition from experimental studies to clinical research.

Conflict of interest: none declared Financial support: none declared

experimental study with silver impregnation methods in the cat. J. Comp. Neurol., 1964 122: 355-367.

6. Blythe Goddardd S., Beuret L. Attention, Balance and Coordination: The A.B.C. of Learning Success, Wiley Blackwell, 2009, Chapter 8, Pages 120-135

7. Tissot R., Monnier M. Functions of the nervous system: Motor and psychomotor functions, Elsevier, 1970 , Chapter 20, Pages 330-390

8. Vinken P.J. Handbook of Clinical Neurology: Disturbances of nervous function. v. 2. Localization in clinical neurology, NorthHolland Publishing Company, 1969, Pages 330-365

9. Dow R.S. The evolution and anatomy of the cerebellum. Biological Reviews, 1942 17: 179-220 
10. Dow R.S. The fiber connections of the posterior parts of the cerebellum in the rat and cat. J. Comp. Neurol., 1936 63: 527-548.

11. Sharma A.C., Kacker S.K. \& Ghosh P. Indian J Otolaryngol (1977) 29: 107.

12. Rorke L.B. The cerebellar medulloblastoma and its relationship to primitive neuroectodermal tumors J Neuropathol Exp Neurol. 1983 Jan;42(1):1-15.

13. Sharma A.C., Kacker S.K. \& Ghosh P. Indian J Otolaryngol (1977) 29: 107.

14. Hart C.W. The Role of Nystagmography in Clinical Diagnosis. Arch Otolaryngol. 1966; 84(6):631-633

15. Gabersek V. Complementary facts on the directional preponderance in nystagmography Rev Neurol (Paris). 1967 Jul; 117(1):293-8.
16. Montandon A., Huguenin S., Luyet M. Experimental study on the modification of diencephalic nystagmus in relation to the position of the head in space Pract Otorhinolaryngol (Basel). 1969; 31(4):204-11

17. Pialoux P., Collard M. Contribution of electronystagmography to otoneurology Rev Neurol (Paris). 1970 Dec; 123(6):447-51

18. Benedict E. Introduction to Electronystagmography, Graduate School, University of Montana, 1985

19. Furman J. Pathophysiology, etiology, and differential diagnosis of vertigo UpToDate, June 2015

20. Furman J., Barton J. Evaluation of the patient with vertigo, UpToDate, June 2015

21. Marianne Dieterich, Thomas Brandt, Functional brain imaging of peripheral and central vestibular disorders. Brain 2008 2538-2552. 